\title{
Marcador Inflamatório, Consumo de Energia, Frutas, Legumes e Verduras em Idosos
}

\author{
Andrieli de Mello da Silva, ${ }^{1}$ Shelly Westphalen Palma, ${ }^{1}$ \\ Mariane Rosa, ${ }^{1}$ Loiva Beatriz Dallepiane ${ }^{2}$
}

\begin{abstract}
RESUMO
Objetivou-se, neste estudo, avaliar a associação do marcador inflamatório (PCRus) com o consumo de energia, frutas, legumes e verduras em idosos. A pesquisa é do tipo quantitativa descritiva e transversal, realizada no munícipio de Palmeira das Missões, Brasil, com idosos com mais de 60 anos, totalizando uma amostra de 43 pessoas. As informações sociodemográficas foram obtidas por meio de entrevista, realizada no domicílio de cada idoso, quando foram orientados a comparecer em jejum de 12 horas, em data e horário definidos, em um laboratório de análises clínicas do município, para coleta de sangue e dos dados antropométricos. O presente estudo apresentou maior frequência para nenhum consumo diário de frutas $(51,2 \%)$ e de legumes e verduras $(58,1 \%)$, consumo de frutas, legumes e verduras (FLV) diário abaixo de três porções $(88,4 \%)$ e Proteína C reativa ultrassensível maior que $0,3 \mathrm{mg} / \mathrm{dL}(51,2 \%)$. Em relação aos dados sociodemográficos, segundo os valores da PCRus, encontrou-se a associação estatisticamente significativa destes com viver sem companheiro $(p=0,033)$. O consumo de energia apresentou-se baixo, bem como a média do consumo de frutas foi inferior a uma porção por dia (0,58 porção/dia) e de legumes e hortaliças $(0,51$ porção/dia). O mesmo baixo consumo foi verificado quando agrupados em FLV, que foi inferior a três porções ao dia, com uma média de 1,12 porções/dia. A maioria dos idosos apresentava os valores do marcador inflamatório PCRus acima de $0,3 \mathrm{mg} / \mathrm{dL}$. Houve relação estatisticamente significativa entre a PCRus somente com a situação conjugal de viver sem companheiro.
\end{abstract}

Palavras-chave: Envelhecimento. Sistema imunitário. Dieta.

INFLAMMATORY MARKER, ENERGY CONSUMPTION, FRUITS, LEGUMES AND GREEN VEGETABLES IN THE ELDERLY ABSTRACT

The objective of this study was to evaluate the association of inflammatory marker (hsCRP) with the consumption of energy, fruits, legumes and vegetables in the elderly. This is a quantitative, descriptive and cross-sectional research, carried out in the city of Palmeira das Missões, Brazil, with the elderly aged over 60 years, totaling a sample of 43 elderly people. The sociodemographic information was obtained through an interview, held in the home of each elderly person, where they were instructed to attend with a 12-hour fasting, on scheduled day and time, a clinical analysis laboratory in the city, for collection of blood samples and anthropometric data. The present study showed higher frequency for no daily consumption of fruits (51.2\%) and legumes and vegetables (58.1\%), daily consumption of fruits, legumes and vegetables (FLV) below three servings (88.4\%) and ultra-sensitive C-Reactive Protein over $0.3 \mathrm{mg} / \mathrm{dl}$ ( $51.2 \%)$. In relation to sociodemographic data, according to the hsCRP values, there was a statistically significant association with living without a partner $(p=0.033)$. The energy consumption was low, as well as the mean consumption of fruits was below one serving per day ( 0.58 serving/day) and of legumes and vegetables $(0.51$ serving/day). The same low consumption occurred when grouped in FLV, which was below three servings per day with an average of 1.12 servings/day. Most of the elderly presented values of hsCRP inflammatory marker above 0.3 $\mathrm{mg} / \mathrm{dl}$. There was a statistically significant relation between hsCRP only with the marital situation of living without a partner. Keywords: Aging. Immune system. Diet.

RECEBIDO EM: 21/4/2020

MODIFICAÇÕES SOLICITADAS EM: 4/9/2020

ACEITO EM: 21/9/2020

\footnotetext{
Universidade Federal de Santa Maria (UFSM) - Campus Palmeira das Missões. Palmeira das Missões/RS, Brasil.

Autora correspondente. Universidade Federal de Santa Maria (UFSM) - Campus Palmeira das Missões. Av. Independência, 3751 - Vista Alegre, $98300-000$. Palmeira das Missões/RS, Brasil. http://lattes.cnpq.br/5570695730349223. https://orcid.org/0000-0003-1331-2882. loiva.dallepiane@hotmail.com
} 


\section{INTRODUÇÃO}

O envelhecimento populacional é um fenômeno que ocorre de modo acentuado em âmbito mundial. No Brasil, as projeções indicam que, até 2030, o número de idosos irá superar o de crianças e adolescentes. Como consequência do envelhecimento populacional, verifica-se um aumento progressivo de doenças crônico-degenerativas e de incapacidades funcionais relacionadas a este processo (MONTEIRO et al., 2020; MIRANDA; MENDES; SILVA, 2016).

A senescência ou envelhecimento constitui-se em um processo dinâmico que envolve mudanças fisiológicas, psicológicas e bioquímicas, com um risco aumentado para doenças crônicas. $O$ envelhecimento é caracterizado por um declínio progressivo das funções corporais e, entre estas, um efeito negativo do envelhecimento é a imunossenescência, a qual se refere à deterioração gradual do sistema imunológico, o que promove um aumento na produção de citocinas pró-inflamatórias e, com isso, favorecem a ocorrência de inflamação crônica em idosos (DE MACEDO et al., 2018).

Estudos revelam que o envelhecimento é acompanhado por uma inflamação em longo prazo denominada de Inflammaging, que está relacionada a eventos adversos em saúde (DE AMORIM et al., 2019). A Inflammaging, inflamação sistêmica crônica e de baixo grau, decorre da diminuição da resposta das funções imunológicas, causando o aumento de mediadores inflamatórios (PIOVEZAN; RIBEIRO, 2016; BAUER, 2019). O marcador inflamatório mais utilizado na prática clínica é a Proteína C Reativa, que, na presença de infecções ou sinais de inflamação, apresenta seus níveis elevados. Por este motivo, é importante identificar previamente o aumento desse marcador, que é um indicativo de fragilidade em idosos (ROZA, 2014).

Um dos fatores influenciadores do marcador inflamatório é a alimentação, uma vez que o baixo consumo de alimentos ricos em fibras e vitaminas como frutas, verduras e legumes, a ingestão elevada de gorduras saturadas, gorduras trans, carboidratos e uma dieta rica em energia podem interferir na saúde do idoso e levar ao desenvolvimento de doenças crônicas, entre elas as doenças inflamatórias (PIOVESAN; RIBEIRO, 2016).

Isso posto, o objetivo deste estudo foi avaliar a associação do marcador inflamatório Proteína C Reativa Ultrassensível (PCRus) com o consumo de energia, fibras, frutas, legumes e verduras em idosos, bem como características sociodemográficas, hábitos de vida e Índice de Massa Corporal (IMC).

\section{METODOLOGIA}

A pesquisa realizada foi do tipo quantitativa, descritiva e transversal. A amostra esteve composta por indivíduos idosos ( $\geq 60$ anos), residentes no município de Palmeira das Missões, RS, sorteados a partir de um cadastro de usuários de três Unidades Básicas de Saúde participantes da Estratégia Saúde da Família (ESF), que compareceram no laboratório para realização de exames bioquímicos, no período de outubro de 2010 a março de 2011, após convite realizado em visita domiciliar, totalizando 43 indivíduos. Foram excluídos os idosos acamados pela dificuldade de aferição do peso e estatura para posterior cálculo do Índice de Massa Corporal.

As variáveis analisadas foram: a) sociodemográficas (idade, sexo, estado civil, escolaridade e renda); b) antropométricas (peso corporal e estatura para cálculo do IMC); c) exames bioquímicos PCRus (Proteína C Reativa ultrassensível); d) consumo de frutas, legumes e verduras (diário); e) consumo de energia (Kcal) e fibras (gramas).

Os dados foram coletados no período de outubro de 2010 a março de 2011. As informações sociodemográficas foram obtidas por meio de entrevista, realizada no domicílio de cada idoso e, nessa ocasião, o participante da pesquisa foi orientado a comparecer em jejum de 12 horas, em data e horário definidos, em um laboratório de análises clínicas do município para coleta de sangue e dos dados antropométricos.

$\mathrm{O}$ peso foi aferido em balança digital portátil, com capacidade até $180 \mathrm{~kg}$. A balança foi posicionada em local plano e os indivíduos tiveram seu peso aferido em posição ereta, sem sapatos, agasalhos ou objetos nos bolsos. A estatura foi verificada com o auxílio de estadiômetro portátil. $\mathrm{O}$ idoso foi posicionado de forma ereta, com os pés descalços, calcanhares unidos, com os braços estendidos ao longo do corpo.

Para a análise do estado nutricional utilizou-se o IMC, calculado a partir da divisão da massa corporal em quilogramas pela estatura em metro elevado ao quadrado $\left(\mathrm{kg} / \mathrm{m}^{2}\right)$. A classificação foi realizada de acordo com os parâmetros de Nutrition Screening Initiative (1994): magreza $\leq 22 \mathrm{~kg} / \mathrm{m}^{2}$; peso normal ou eutrófico de 22 a $27 \mathrm{~kg} / \mathrm{m}^{2}$ e excesso de massa corporal $\geq 27 \mathrm{~kg} / \mathrm{m}^{2}$.

A avaliação do consumo diário de frutas, legumes e verduras foi feita mediante a aplicação de um questionário de frequência alimentar e para fins de análise foram consideradas as variáveis de consumo diário de frutas (F); legumes e verduras (LV) e do agrupamento de frutas verduras e legumes (FLV). $\mathrm{O}$ consumo de frutas e 
o de legumes e verduras foram categorizadas em: nenhuma porção/dia, uma porção/dia, duas porções ao dia (porção máxima identificada na pesquisa), e o consumo de FLV foi categorizado em $<3$ porções e $\geq 3$ porções.

Para o cálculo do consumo de energia e fibras foi realizado o recordatório alimentar de 24 horas (R24h). O cálculo do R24 horas foi feito utilizando o Software Avanutri on-line. $\mathrm{O}$ consumo de energia foi agrupado em três categorias: a) <1000 Kcal; b) 1000-1499 Kcal; c) $\geq 1500 \mathrm{Kcal}$ e o de fibras em adequado ou inadequado.

Como marcador inflamatório foi utilizado o exame de PCRus. O ponto de corte foi $0,3 \mathrm{mg} / \mathrm{dL}$, pois somente os métodos ultrassensíveis captam tais valores. Por ser um método de custo elevado, foi possível de ser realizado com apenas os 43 idosos que compareceram em jejum no laboratório no período determinado anteriormente. Foram consideradas, então, as categorias $\leq 0,3$ (adequado) e > 0,3 (inadequado).

Os dados foram analisados utilizando-se o software estatístico SPSS (Statistical Package for the Social Sciences ${ }^{\circledR}$ ) versão 18.0. A metodologia de análise foi a estatística descritiva e teste de Qui-quadrado ao nível de significância de $5 \%$.

O presente estudo é um recorte de um projeto maior denominado "Situação alimentar e nutricional da população idosa de Palmeira das Missões, RS" e, portanto, seguiu as recomendações da Resolução 196/96 do CNS e foi submetido ao Comitê de Ética em Pesquisa, da Universidade Federal de Santa Maria, no ano de 2010, sob número 23081.009908/2010-10. Após a concordância, os participantes da pesquisa assinaram um Termo de Consentimento Livre e Esclarecido - TCLE.

\section{RESULTADOS}

Participaram do estudo 43 idosos, a maioria do sexo feminino $(58,1 \%)$, de 60 a 69 anos $(67,4 \%)$, com companheiro $(86,0 \%)$, escolaridade abaixo de 5 anos $(86,0 \%)$, renda menor que 2 salários mínimos $(79,1 \%)$, IMC em sobrepeso (60,5 \%), não tabagista $(90,7 \%)$, sem consumo de bebida alcoólica $(74,4 \%)$, não praticante de atividades físicas $(69,8$ $\%)$, fazendo uso de medicamentos $(74,4 \%)<5$ por dia $(62,8 \%)$ (Tabela 1).

De acordo com a Tabela 2, as maiores frequências observadas foram do consumo de energia, entre 1.000 a $1.499 \mathrm{Kcal} / \mathrm{dia}(44,2 \%)$, consumo de fibras inadequado (93\%), nenhum consumo diário de frutas $(51,2 \%)$ e de legumes e verduras $(58,1 \%)$, consumo de frutas, legumes e verduras agrupadas (FLV) diário foi menor de 3 porções $(88,4 \%)$ e Proteína C reativa ultrassensível maior que $0,3 \mathrm{mg} / \mathrm{dL}(51,2 \%)$.
Tabela 1 - Características sociodemográficas, hábitos de vida e IMC de idosos. Palmeiras das Missões, RS, 2011

\begin{tabular}{|c|c|c|}
\hline Variável & $\mathrm{n}$ - & $\%$ \\
\hline \multicolumn{3}{|l|}{ Gênero } \\
\hline Feminino & 25 & 58,1 \\
\hline Masculino & 18 & 41,9 \\
\hline \multicolumn{3}{|l|}{ Idade } \\
\hline 60-69 anos & 29 & 67,4 \\
\hline 70-79 anos & 11 & 25,6 \\
\hline 80 anos ou mais & 3 & 7 \\
\hline \multicolumn{3}{|l|}{ Situação conjugal } \\
\hline Sem companheiro & 15 & 14 \\
\hline Com companheiro & 28 & 86 \\
\hline \multicolumn{3}{|l|}{ Escolaridade } \\
\hline$<5$ anos & 37 & 86 \\
\hline$\geq 5$ anos & 6 & 14 \\
\hline \multicolumn{3}{|l|}{ Renda } \\
\hline$\leq 2$ salários mínimos & 34 & 79,1 \\
\hline > 2 salários mínimos & 9 & 20,9 \\
\hline \multicolumn{3}{|l|}{ IMC* } \\
\hline Magreza & 1 & 2,3 \\
\hline Eutrofia & 16 & 37,2 \\
\hline Excesso de peso & 26 & 60,5 \\
\hline \multicolumn{3}{|l|}{ Tabagismo } \\
\hline Sim & 4 & 9,3 \\
\hline Não & 39 & 90,7 \\
\hline \multicolumn{3}{|l|}{ Bebida alcoólica } \\
\hline Sim & 11 & 25,6 \\
\hline Não & 32 & 74,4 \\
\hline \multicolumn{3}{|l|}{ Faz Atividade Física } \\
\hline Sim & 13 & 30,2 \\
\hline Não & 30 & 69,8 \\
\hline \multicolumn{3}{|l|}{ Uso de medicação } \\
\hline Sim & 32 & 74,4 \\
\hline Não & 11 & 25,6 \\
\hline \multicolumn{3}{|l|}{ № Medicamentos/dia } \\
\hline$<5$ & 27 & 62,8 \\
\hline$\geq 5$ & 5 & 11,6 \\
\hline
\end{tabular}

*IMC= Índice de Massa Corporal

Fonte: Elaborada pelos autores.

A Tabela 3 apresenta a relação dos dados sociodemográficos, hábitos de vida e IMC segundo os valores da PCRus. Foi encontrada associação estatisticamente significativa entre a situação conjugal viver sem companheiro ( $p=0,033$ ) e a PCRus. Embora não encontrados resultados significativos, a maior frequência de PCRus inadequada foi encontrada no sexo feminino $(p=0,172), 80$ anos ou mais $(p=0,804)$, escolaridade abaixo de 5 anos ( $p=0,951)$, renda maior que 2 salários mínimos ( $p=0,767)$, IMC e excesso de peso $(p=0,398)$, sem hábito de tabagismo $(\mathrm{p}=0,961)$ e de bebida alcoólica $(p=0,661)$, praticante de atividade física $(p=0,817)$, sem uso de medicamentos $(p=0,337)$ e consumo de medicamento menor que 5 por dia $(p=0,737)$. 
Tabela 2 - Média e frequência do consumo de energia, fibras, frutas, legumes, verduras e dosagem de Proteína C Reativa ultrassensível de idosos. Palmeiras das Missões, RS, 2011

\begin{tabular}{|c|c|c|}
\hline Variável & $\mathrm{n}$ 은 & $\%$ \\
\hline \multicolumn{3}{|c|}{ Consumo de energia - Kcal (Média=1286,1士481,1) } \\
\hline$<1000$ & 13 & 30,2 \\
\hline 1000-1499 & 19 & 44,2 \\
\hline$\geq 1500$ & 11 & 25,6 \\
\hline \multicolumn{3}{|c|}{ Consumo de fibras - Gramas (Média=13,07士7,30) } \\
\hline Adequado & 3 & 7 \\
\hline Inadequado & 40 & 93 \\
\hline \multicolumn{3}{|c|}{ 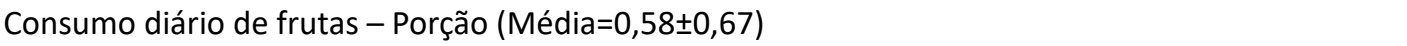 } \\
\hline Nenhuma & 22 & 51,2 \\
\hline Uma & 17 & 39,5 \\
\hline Duas & 4 & 9,3 \\
\hline \multicolumn{3}{|c|}{ Consumo diário de legumes e verduras - Porção (Média=0,51 $\pm 0,67$ ) } \\
\hline Nenhuma & 25 & 58,1 \\
\hline Uma & 14 & 32,6 \\
\hline Duas & 4 & 9,3 \\
\hline \multicolumn{3}{|c|}{ 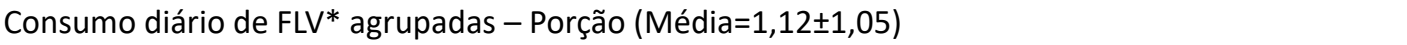 } \\
\hline$<3$ porções & 38 & 88,4 \\
\hline$\geq 3$ porções & 5 & 11,8 \\
\hline \multicolumn{3}{|c|}{ PCRus (mg/dL)** } \\
\hline$\leq 0,3$ & 21 & 48,8 \\
\hline$>0,3$ & 22 & 51,2 \\
\hline
\end{tabular}

Tabela 3 - Relação dos dados sociodemográficos, hábitos de vida e IMC com a PCRus de idosos. Palmeiras das Missões, RS, 2011

\begin{tabular}{|c|c|c|c|c|}
\hline \multirow{3}{*}{ Variável } & \multicolumn{3}{|c|}{ PCRus } & \multirow[t]{3}{*}{ p* } \\
\hline & $\begin{array}{l}\text { Adequado } \\
(\leq 0,3 \mathrm{mg} / \mathrm{dL})\end{array}$ & $\begin{array}{l}\text { Inadequado } \\
(>0,3 \mathrm{mg} / \mathrm{dL})\end{array}$ & Total & \\
\hline & $\mathrm{n}(\%)$ & $\mathrm{n}(\%)$ & $\mathrm{n}(\%)$ & \\
\hline Sexo & & & & 0,172 \\
\hline Feminino & $10(40)$ & $15(60)$ & $25(100)$ & \\
\hline Masculino & $11(61,1)$ & $7(38,9)$ & $18(100)$ & \\
\hline Total & $21(48,8)$ & $22(51,2)$ & $43(100)$ & \\
\hline Idade & & & & 0,804 \\
\hline 60-69 anos & $14(48,3)$ & $15(51,7)$ & $29(100)$ & \\
\hline 70-79 anos & $6(54,5)$ & $5(45,5)$ & $11(100)$ & \\
\hline 80 anos ou mais & $1(33,3)$ & $2(66,7)$ & $3(100)$ & \\
\hline Total & $21(48,8)$ & $22(51,2)$ & $43(100)$ & \\
\hline Situação conjugal* & & & & 0,033 \\
\hline Sem companheiro & $4(26,7)$ & $11(73,3)$ & $15(100)$ & \\
\hline Com companheiro & $17(60,7)$ & $11(39,3)$ & $28(100)$ & \\
\hline Total & $21(48,8)$ & $22(51,2)$ & $43(100)$ & \\
\hline Escolaridade & & & & 0,951 \\
\hline$<5$ anos & $18(48,6)$ & $19(51,4)$ & 37 (100) & \\
\hline$\geq 5$ anos & $3(50,0)$ & $3(50)$ & $6(100)$ & \\
\hline Total & $21(48,8)$ & $22(51,2)$ & $43(100)$ & \\
\hline Renda & & & & 0,767 \\
\hline$\leq 2$ salários mínimos & $17(50,0)$ & $17(50)$ & $34(100)$ & \\
\hline
\end{tabular}




\begin{tabular}{|c|c|c|c|c|}
\hline > 2 salários mínimos & $4(44,4)$ & $5(55,6)$ & $9(100)$ & \\
\hline Total & $21(48,8)$ & $22(51,2)$ & $43(100)$ & \\
\hline IMC & & & & 0,398 \\
\hline Baixo peso & $1(100)$ & $0(0,00)$ & $1(100)$ & \\
\hline Eutrofia & $9(56,3)$ & $7(43,8)$ & $16(100)$ & \\
\hline Excesso de peso & $11(42,3)$ & $15(57,7)$ & $26(100)$ & \\
\hline Total & $21(48,8)$ & $22(51,2)$ & $43(100)$ & \\
\hline Hábito de fumar & & & & 0,961 \\
\hline Sim & $2(50,0)$ & $2(50)$ & $4(100)$ & \\
\hline Não & $19(48,7)$ & $20(51,3)$ & $39(100)$ & \\
\hline Total & $21(48,8)$ & $22(51,2)$ & $43(100)$ & \\
\hline Bebida alcoólica & & & & 0,661 \\
\hline Sim & $6(54,5)$ & $5(45,5)$ & $11(100)$ & \\
\hline Não & $15(46,9)$ & $17(53,1)$ & $32(100)$ & \\
\hline Total & $21(48,8)$ & $22(51,2)$ & $43(100)$ & \\
\hline Faz atividade física & & & & 0,817 \\
\hline $\operatorname{Sim}$ & $6(46,2)$ & $7(53,8)$ & $13(100)$ & \\
\hline Não & $15(50,0)$ & $15(50)$ & $30(100)$ & \\
\hline Total & $21(48,8)$ & $22(51,2)$ & $43(100)$ & \\
\hline Uso de medicamentos & & & & 0,337 \\
\hline $\operatorname{Sim}$ & $17(53,1)$ & $15(46,9)$ & $32(100)$ & \\
\hline Não & $4(36,4)$ & $7(63,6)$ & $11(100)$ & \\
\hline Total & $21(48,8)$ & $22(51,2)$ & $43(100)$ & \\
\hline $\mathrm{N} \cong$ Medicamentos/dia & & & & 0,737 \\
\hline$<5$ & $14(51,9)$ & $13(48,1)$ & $27(100)$ & \\
\hline$\geq 5$ & $3(60,0)$ & $2(40)$ & $5(100)$ & \\
\hline Total & $17(53,1)$ & $15(46,9)$ & $32(100)$ & \\
\hline
\end{tabular}

${ }^{*} \mathrm{p}<0,05=$ significativo

Fonte: Elaborada pelos autores.

De acordo com a Tabela 4, a maior frequência da PCRus inadequada foi observada no consumo de energia abaixo de $1.000 \mathrm{kcal}(p=0,071)$, fibras inadequadas $(p=0,522)$, nenhum consumo diário de frutas $(p=0,540)$, consumo de uma porção de legumes e verduras $(p=0,860)$, frutas e legumes e verduras agrupados diário $\leq 3$ porções $(p=0,595)$, embora nenhum apresente associação significativa.

Tabela 4- Relação do consumo de energia, fibras, frutas e hortaliças com a PCRus de idosos. Palmeiras das Missões, RS, 2011

\begin{tabular}{|c|c|c|c|c|}
\hline \multirow[t]{3}{*}{ Variável } & \multicolumn{2}{|c|}{ PCRus } & \multirow[b]{2}{*}{ Total $(n=43)$} & \multirow[b]{2}{*}{$\mathrm{P}$} \\
\hline & $\begin{array}{c}\text { Adequado } \\
(\leq 0,3 \mathrm{mg} / \mathrm{dL})\end{array}$ & $\begin{array}{l}\text { Inadequado } \\
(>0,3 \mathrm{mg} / \mathrm{dL})\end{array}$ & & \\
\hline & $\mathrm{n}$ - $(\%)$ & $\mathrm{n}$ - $(\%)$ & $\mathrm{n}-(\%)$ & \\
\hline Consumo de energia (kcal) & & & & 0,071 \\
\hline$<1.000$ & $4(30,8)$ & $9(69,2)$ & $13(100)$ & \\
\hline $1.000-1499$ & $13(68,4)$ & $6(31,6)$ & $19(100)$ & \\
\hline$\geq 1.500$ & $4(36,4)$ & $7(63,6)$ & $11(100)$ & \\
\hline Total & $21(48,8)$ & $22(51,2)$ & $43(100)$ & \\
\hline Consumo de fibras & & & & 0,522 \\
\hline Adequado & $2(66,7)$ & $1(33,3)$ & $3(100)$ & \\
\hline Inadequado & $19(47,5)$ & $21(52,5)$ & $40(100)$ & \\
\hline Total & $21(48,8)$ & $22(51,2)$ & $43(100)$ & \\
\hline Consumo diário de frutas & & & & 0,540 \\
\hline Nenhuma & $9(40,9)$ & $13(59,1)$ & $22(100)$ & \\
\hline
\end{tabular}




\begin{tabular}{lccc} 
Uma porção & $10(58,8)$ & $7(49,2)$ & $17(100)$ \\
Duas porções & $2(50)$ & $2(50)$ & $4(100)$ \\
Total & $21(48,8)$ & $22(51,2)$ & $43(100)$ \\
Consumo diário de legumes e verduras & & & 0,860 \\
Nenhuma & $13(52)$ & $12(48)$ & $25(100)$ \\
Uma porção & $6(42,9)$ & $8(57,1)$ & $14(100)$ \\
Duas porções & $2(50)$ & $2(50)$ & $4(100)$ \\
Total & $21(48,8)$ & $22(51,2)$ & $43(100)$ \\
Consumo diário de FLV* agrupados & & & $38(100)$ \\
$\leq 3$ porções & $18(47,4)$ & $20(52,6)$ & $5(100)$ \\
$>3$ porções & $3(60)$ & $2(40)$ & $43(100)$ \\
Total & $21(48,8)$ & $22(51,2)$ & \\
\hline
\end{tabular}

*FLV= Frutas, legumes e verduras

\section{DISCUSSÃO}

No presente estudo encontrou-se uma baixa frequência de ingestão média diária de frutas, legumes/verduras e FLV, respectivamente, de 0,58 $\pm 0,66$, $0,51 \pm 0,668$ e $1,12 \pm 1,051$ porções, todas abaixo da recomendação. As frutas, legumes e verduras são alimentos ricos em fibras, vitaminas e minerais e devem ser consumidos diariamente, pois contribuem na meIhoria da saúde e diminuem o risco de desenvolver doenças. Segundo o guia alimentar para a população brasileira, o consumo de frutas, verduras e legumes é preventivo contra doenças do coração e alguns tipos de cânceres, por isso recomenda-se o consumo diário de três porções de frutas e três de legumes e verduras (BRASIL, 2014).

Para manter um estado saudável durante o processo de envelhecimento, é aconselhável incentivar as próprias defesas anti-inflamatórias. Entre um grande painel de recomendações alimentares, a dieta mediterrânea é reconhecida como uma estratégia confiável para neutralizar o processo de inflamação devido ao seu alto conteúdo de compostos polifenólicos, principalmente flavonoides, além de vitaminas, gorduras boas e carboidratos complexos (MARTUCCl et al., 2017). As frutas cítricas e seus sucos são uma das principais fontes de flavonoides na dieta mediterrânea, pelos efeitos protetores contra o estresse oxidativo e inflamação (FERLAZZO et al., 2016), infecções microbianas (CIRMI et al., 2016), cardiovasculares (TESTAl; CALDERONE, 2017) e doenças neurodegenerativas (CITRARO et al., 2016) e alguns tipos de câncer (CIRMI, 2018).

Quando comparado o consumo de FLV diário com outros estudos, os achados foram semelhantes, porém com amostras maiores. Em estudo realizado por Silveira et al. (2015), em Goiânia-GO, onde foram avaliados 416 idosos com idade $\geq 60$ anos, o consumo de frutas e hortaliças diário foi de apenas $16,7 \%$ da população avaliada. Em outro estudo realizado com adultos e idosos por Oliveira et al. (2015), em Minas Gerais, com uma amostra de 1.255 sujeitos, avaliou-se que a ingestão de frutas e hortaliças foi inadequada em $77,5 \%$ dos participantes.

Em relação ao consumo de energia (kcal), a média do consumo diário foi de $1.286,1 \pm 481,1$ consumo considerado inadequado. $O$ Institute of Medicine (2002) preconiza para homens um valor energético total (VET) de $3.067 \mathrm{Kcal}$ e para mulheres $2.403 \mathrm{Kcal}$, subtraindo deste valor $10 \mathrm{Kcal} /$ dia para homens e $7 \mathrm{Kcal} /$ dia para as mulheres para cada ano acima de 19 anos de idade.

Em estudo realizado por Silva et al. (2016) em um município no Sul do Brasil com uma amostra de 424 idosos, dos quais $84,4 \%$ com idade inferior a 80 anos (idosos jovens) e $15,6 \%$ com 80 anos e mais (idosos longevos), a ingestão de energia foi insuficiente em $92,7 \%$ entre os idosos jovens e de $84,8 \%$ entre os idosos longevos. Em estudo realizado por Venturini et al. (2015), em Porto Alegre, em um projeto de pesquisa com 427 idosos, constatou-se uma média do consumo de energia de $1.564,0 \pm 553,9$ no gênero masculino e $1.330,8 \pm 428,7$ no gênero feminino.

Ao determinar o consumo diário de fibras, a média foi de $13,07 \pm 7,30$, avaliado como inadequado. A recomendação do consumo diário de fibras pode variar conforme a idade, o gênero e o consumo de energia, sendo recomendado em torno de $14 \mathrm{~g}$ de fibras para cada $1.000 \mathrm{Kcal}$ consumidas. No envelhecimento, a ingestão desse nutriente é de grande importância, pois pode trazer benefícios à saúde, dado que seu consumo adequado pode reduzir o risco do desenvolvimento de doenças crônicas não transmissíveis (BERNAUD; RODRIGUES, 2013). Silva et al. (2019) 
realizaram um estudo em Campinas, em que foram entrevistados 1.509 idosos, e constataram que 90,1\% tinham consumo de fibras inadequado, com média de ingestão diária de 13,5 g (SILVA et al., 2019).

No presente estudo a PCRus teve associação significativa somente com a situação conjugal viver sem companheiro $(p=0,033)$. Viver sem companheiro mostrou-se estar relacionado à fragilidade em um estudo realizado por Carneiro et al. (2017), que avaliou a prevalência e fatores associados à fragilidade em 360 idosos com idade igual ou superior a 65 anos e apresentou significância estatística com o estado civil viver sem companheiro. Corroborando esta hipótese da vulnerabilidade do idoso que vive sem companheiro, o estudo de Campos et al. (2016) com idosos octagenários de Sete Lagoas, Minas Gerais, indicou que o envelhecimento saudável esteve significativamente associado com a condição de ser casado $(p=0,015)$ e não ter cuidador $(p=0,014)$.

O estudo apresenta como limitação o próprio delineamento do tipo transversal e o fato de ter uma amostra pequena, em razão do custo elevado de cada exame de PCRus, sem financiamento externo.

\section{CONCLUSÃO}

Os idosos apresentaram baixa ingestão energética, bem como a média do consumo de frutas foi inferior a uma porção por dia (0,58 porção/dia) e de legumes e hortaliças (0,51 porção/dia). O mesmo baixo consumo foi verificado quando agrupados em FLV, que foi inferior a três porções ao dia, com uma média de 1,12 porções/dia. A maioria dos idosos apresentava os valores do marcador inflamatório PCRus acima de $0,3 \mathrm{mg} / \mathrm{dL}$. Somente houve relação estatisticamente significativa entre a PCRus e situação conjugal de viver sem companheiro.

\section{REFERÊNCIAS}

BAUER, E. M. Imunosenescência: envelhecimento do sistema imune. 1. ed. Porto Alegre: Edipucrs, 2019.

BERNAUD, R. S. F.; RODRIGUES, C. T. Fibra alimentar - ingestão adequada e efeitos sobre a saúde do metabolismo. Arquivos Brasileiros de Endocrinologia \& Metabologia, v. 57, n. 6, p. 397-405, 2013.

BRASIL. Guia alimentar para a população brasileira. 2. ed. Brasília, DF: Ministério da Saúde, 2014.

CAMPOS, A. C. V. et al. Healthy aging profile in octogenarians in Brazil. Rev. Latino-Am. Enfermagem, 24, p. e2724, 2016. Disponível em: https://www.scielo.br/pdf/rlae/v24/ pt_0104-1169-rlae-24-02724.pdf. Acesso em: 15 set. 2020. DOI: http://dx.doi.org/10.1590/1518-8345.0694.2724.
CARNEIRO, A. J. et al. Fragilidade em idosos: prevalência e fatores associados. Rev. Brasileira de Enfermagem, v. 70, n. 4, p. 780-785, 2017.

CIRMI, S. et al. Anti-infective potential of Citrus bergamia Risso et Poiteau (bergamot) derivatives: a systematic review. Phytother Res, v. 30, n. 9, p. 1.404-1.411, 2016.

CIRMI, S. et al. Citrus fruits intake and oral cancer risk: a systematic review and metaanalysis. Pharmacol. Res., v. 133, p. 187-194, 2018.

CITRARO, R. et al. The Anticonvulsant activity of a flavonoid-rich extract from orange juice involves both NMDA and GABA-benzodiazepine receptor complexes. Molecules, v. 21, n. 9, p. 1.261-1.274, 2016.

DE AMORIM, J. S. C. et al. Marcadores inflamatórios e ocorrência de quedas: coorte de idosos de Bambuí. Rev. Saúde Pública, v. 53, n. 35, 2019.

DE MACEDO, B. G. et al. Associação entre mediadores inflamatórios, força de preensão manual e mobilidade em idosos da comunidade. Fisioterapia em Movimento, Curitiba, v. 31, p. e003132, 2018.

FERLAZZO, N. et al. Anti-inflammatory activity of Citrus bergamia derivatives: where do we stand? Molecules, v. 21, n. 10, p. 1.273-1.283, 2016.

INSTITUTE OF MEDICINE. Food and Nutrition Board. Dietary reference intakes for energy, carbohydrate, fiber, fat, fatty acids, cholesterol, protein and amino acids. Journal of the American Dietetic Association, Washington, DC: National Academy Press, p. 697-736, Nov. 2002.

MARTUCCI, M. et al. Mediterranean diet and inflammaging within the hormesis paradigm. Nutr. Rev., v. 75, n. 6, p. 442455, 2017.

MIRANDA, G. M. D.; MENDES, A. C. G.; SILVA, A. L. A. O envelhecimento populacional brasileiro: desafios e consequências sociais atuais e futuras Rev. Bras. Geriatr. Gerontol., v. 19, n. 3, p. 507-519, 2016.

MONTEIRO, I. O. et al. A saúde do idoso na graduação em fisioterapia no Brasil: um estudo transversal. Fisioterapia $e$ Pesquisa, São Paulo, v. 27, n. 1, p. 93-99, 2020.

OLIVEIRA, S. M. et al. Consumo de frutas e hortaliças e as condições de saúde de homens e mulheres atendidos na atenção primária à saúde. Rev. Ciência \& Saúde Coletiva, v. 20, n. 8, p. 2,313-2,322, 2015.

PIOVEZAN, R; RIBEIRO S. Inflammaging: inflamação sistêmica e de baixo grau decorrente do envelhecimento. Rio de Janeiro: Sociedade Brasileira de Geriatria e Gerontologia, 2016. Disponível em: https://sbgg.org.br//wp-content/ uploads/2014/11/18761A-Separata_Inflammaging.pdf. Acesso em: 3 dez. 2018.

ROZA, B. L. Fragilidade em idosos hospitalizados determinada por marcadores inflamatórios: prevalência e fatores associados. 2014. 95 p. Dissertação (Mestrado em atenção à saúde) - Universidade Federal do Triângulo Mineiro, Uberaba, 2014.

SILVA, M. G. et al. Elevada prevalência de inadequação do consumo de fibras alimentares em idosos e fatores associados: um estudo de base populacional. Rev. Bras. Epidemio- 
logia, v. 22, p. e190044, 2019. Disponível em: http://www. scielo.br/pdf/rbepid/v22/1980-5497-rbepid-22-e190044. pdf. Acesso em: 13 nov. 2019.

SILVA, P. F. et al. Compliance in food consumption of young and long-lived elderly of a city in southern Brazil. Rev. Nutrición Hospitalaria, v. 33, n. 1, p. 37-42, 2016.

SILVEIRA, A. E. et al. Baixo consumo de frutas, verduras e legumes: fatores associados em idosos em capital no Centro-Oeste do Brasil. Rev. Ciência \& Saúde Coletiva, v. 20, n. 12, p. 3.689-3.699, 2015.

TESTAI, L.; CALDERONE, V. Nutraceutical value of citrus flavanones and their implications in cardiovascular disease. Nutrients, v. 9, n. 5, p. 502-514, 2017.

VENTURINI, D. C. et al. Consumo de nutrientes em idosos residentes em Porto Alegre (RS), Brasil: um estudo de base populacional. Rev. Ciência \& Saúde Coletiva, v. 20, n. 12, p. 3.701-3.711, 2015. 